\title{
RANKing C-Jun in osteoclast development
}

\author{
Steven L. Teitelbaum
}

\author{
Department of Pathology, Washington University School of Medicine, St. Louis, Missouri, USA.
}

\begin{abstract}
Pathological bone loss always reflects enhanced net osteoclastic activity. Recognition and binding of the receptor activator of NF- $K B$ (RANK) by RANK ligand (RANKL) is the key osteoclastogenic event, and the signaling cascades induced by this reaction therefore contain potential anti-osteoporosis therapeutic targets. A study reported in this issue of the JCI documents that a pivotal component of RANKL/RANK-mediated osteoclast recruitment involves sequential induction of the transcription factors c-Jun and nuclear factor of activated T cells 2 (see the related article beginning on page 475).
\end{abstract}

\section{What is an osteoclast?}

Osteoporosis always represents an imbalance in favor of osteoclast-mediated bone resorption relative to the bone-forming capacity of osteoblasts. In some conditions of accelerated skeletal loss, such as Paget disease, osteoclasts are greatly enlarged, which enhances the individual cell's resorptive activity. Most osteopenic disorders, however, develop as a consequence of accelerated bone degradation due to increased osteoclast number. Thus, inhibition of osteoclast formation by estrogen supplementation, or osteoclast function by bisphosphonate administration, has been the most effective means of arresting pathological bone loss. However, both forms of therapy are not without drawbacks, and new anti-resorptive targets are rapidly emerging. In this issue of the JCI, Ikeda et al. elegantly show that the JNK-1-activated c-Jun signaling pathway is key to osteoclastic bone resorption (1). The authors find that mice bearing a dominant-negative osteoclast-specific $c$-Jun transgene have impaired osteoclastogenesis and, because of failed bone resorption, develop increased bone mass in the form of osteopetrosis. Importantly, the failure of dominant-negative $c-J u n$ transgenic mice to generate osteoclasts is due to arrested activation and expression of members of the nuclear factor of activated $\mathrm{T}$ cells (NFAT) family of transcription factors in osteoclast precursors.

Nonstandard abbreviations used: activator protein1 (AP-1); MAPK kinase 7 (MKK7); nuclear factor of activated T cells (NFAT); receptor activator of NF-KB (RANK); RANK ligand (RANKL); TNF receptor-associated factor 6 (TRAF6)

Conflict of interest: S.L. Teitelbaum is a scientific founder of Auxeris Therapeutics Inc., which develops anti-osteoporosis drugs.

Citation for this article:

J. Clin. Invest. 114:463-465 (2004).

doi:10.1172/JCI200422644.
The osteoclast is a huge cell whose phenotype is characterized by its capacity to attach to bone and polarize its resorptive machinery toward the cell's interface with mineralized matrix (2). It is a terminally differentiated polykaryon whose multinucleation is a manifestation of fusion rather than endomitosis, and it is the unique resorbing cell of the skeleton.

\section{RANK ligand is key}

Donald Walker, in the 1970s, performed the first meaningful experiments with osteopetrotic mice, which have, from that time forward, served as the essential resource for understanding how osteoclasts are born, resorb bone, and die. Using cross-circulation and marrow cell transplantation, Walker established that the enigmatic polykaryon is of hematopoietic origin (3). A decade later, Suda's group documented that the osteoclast can be generated, in vitro, from mononuclear members of the monocyte/ macrophage family, thus establishing their identity as osteoclast precursors (4). Curiously, however, Suda was unable to generate osteoclasts unless their mononuclear progenitors were in physical contact with marrow-derived mesenchymal stromal cells, including osteoblasts. Discovery of the molecule expressed on the surface of stromal cells that mediates commitment of macrophages to the bone resorptive phenotype remained the principal enigma of osteoclast biology until 1998, when this key osteoclastogenic moiety was identified as receptor activator of NF-KB (RANK) ligand (RANKL) $(5,6)$. RANKL is a member of the TNF superfamily whose crystal structure reveals unique components that enable it to activate its osteoclastogenic receptor, RANK, on osteoclast precursors (7) (Figure 1). While other cytokines, particularly TNF- $\alpha$, may be profoundly synergistic, only activated RANKL/RANK signaling suffices and is essential for osteoclast differentiation $(8,9)$.

The discovery of the osteoclastogenic capacity of RANKL enables generation of large numbers of essentially pure populations of bona fide osteoclasts in vitro, which in turn permits meaningful biochemical and molecular definition of this cell. We now know that RANKL is a homotrimer (7), which not only promotes differentiation of osteoclast precursors but also activates the mature bone-degrading polykaryon (10). Thus, RANKL and RANK, as well as their intracellular signaling pathway, are presently the most promising antiresorptive therapeutic targets.

\section{The osteoclast needs}

\section{TRAF6, Fos, and Jun}

The importance of activator protein-1 (AP-1) transcription factors, specifically dimers of the Fos and Jun families of proteins, in the osteoclastogenic process was first documented in the laboratory of Erwin Wagner, wherein c-Fos knockout mice were shown to be osteopetrotic due to a complete absence of osteoclasts (11). Consequently, the discovery of RANKL as the key osteoclastogenic cytokine prompted interest in the mechanisms by which RANK activation regulates AP-1 transcription factors.

Just why RANKL is unique among TNF superfamily members in its capacity to induce osteoclast differentiation is still unresolved but probably involves its interaction with TNF receptor-associated factor 6 (TRAF6) $(12,13)$. Other RANKL-stimulated intracellular signaling molecules essential to the osteoclast phenotype include the p50/ p52 NF-KB subunits and the PI3K/AKT axis. Similarly, the MAPKs extracellular regulated kinases 1 and 2 and p38 are required for osteoclast differentiation or function.

Association of RANKL and TRAF6 activates all key events involving AP-1-mediated transcription of osteoclast specific genes. Expression of the $c$-Fos gene in 393T cells requires TRAF 6 , but whether the same holds true in osteoclasts is unresolved. On the other hand, c-Jun is clearly RANKL 


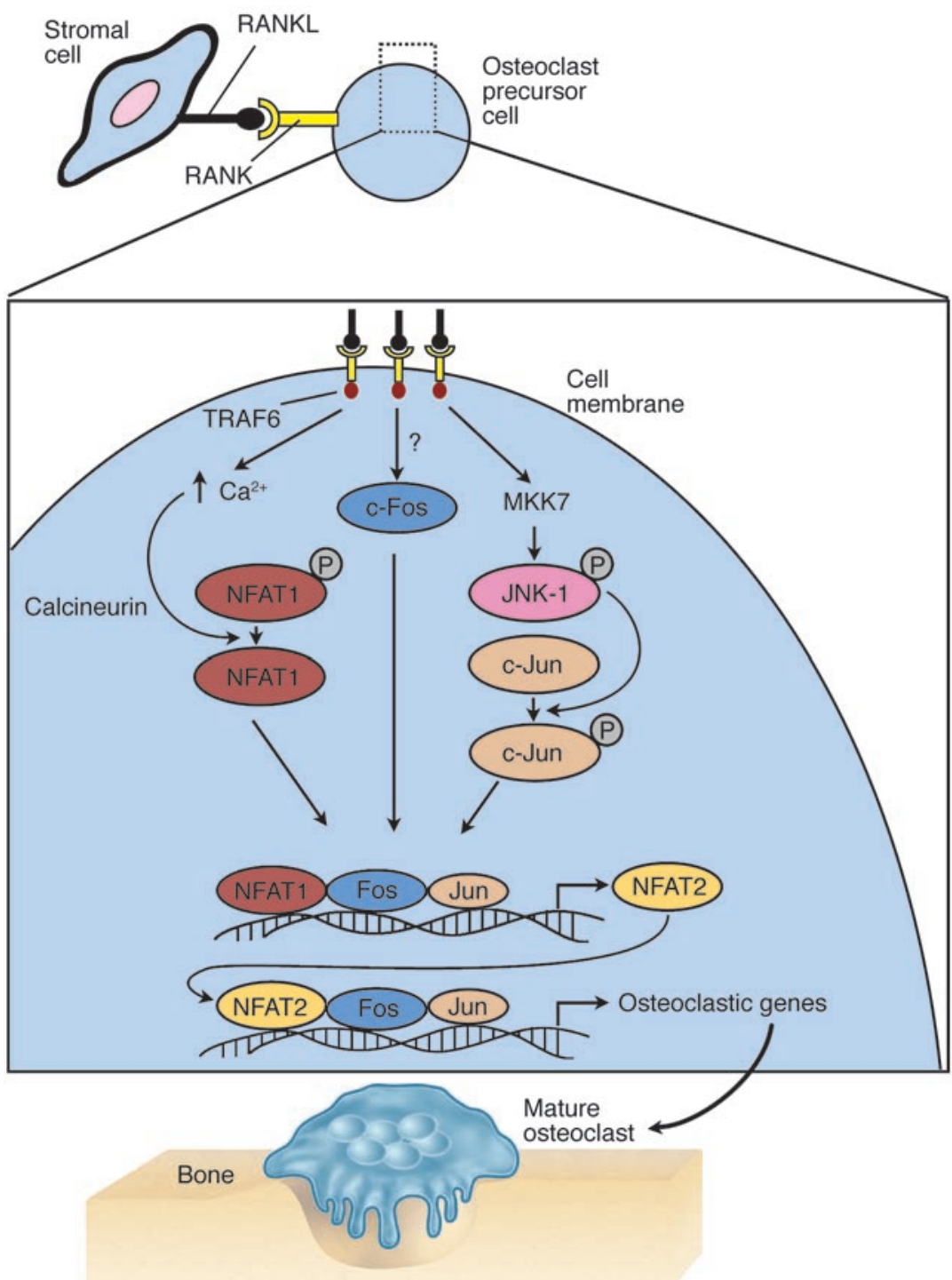

Figure 1

The AP-1/NFAT transcription complex mediates osteoclast precursor differentiation. Osteoclastogenesis is initiated by RANKL occupying RANK on the surface of osteoclast precursors. Subsequent recruitment of TRAF6 initiates the 3 depicted signaling cascades, in addition to other pathways not shown here. Phosphorylation (P) activates c-Jun in an MKK7/JNK-1-dependent manner, and NFAT1 is activated by dephosphorylation via calcium-mediated induction of calcineurin. RANKL/RANK also induces c-Fos expression by an incompletely understood mechanism. NFAT1 partners with the AP-1 proteins of the Fos/Jun families to transactivate the NFAT2 gene, the product of which forms a similar ternary transcription complex on osteoclastic genes eventuating in appearance of the mature osteoclast phenotype.

activated via TRAF6 by a process involving JNK1 but not JNK2 (14). c-Jun is not the only member of its family to regulate osteoclastogenesis, as Jun-B-deficient mice also have arrested bone resorption (15).

When transcriptionally active, c-Jun associates with members of the Fos family of transcription factors, a number of which - such as Fra-1, itself a c-Fos target - may substitute for c-Fos in the osteoclastogenic process (16). Although c-Jun also homodi-

does not rescue the $c$-Fos-/- osteopetrotic phenotype indicates that c-Jun is necessary, but not sufficient, to transactivate osteoclast specific genes.

\section{NFAT and AP-1}

In 2002 Takayanagi et al. identified NFAT2 by genome-wide screening as the predominant gene activated in osteoclast precursors under the influence of RANKL and docu- mented the transcription factor's essential role in osteoclastogenesis (17). RANK occupancy mobilizes intracellular calcium, a requisite for calcineurin-mediated NFAT activation (Figure 1). Moreover, RANKL not only induces the transcription factor's expression but facilitates its nuclear translocation, where NFAT binds to its DNA response element via a ternary complex with AP-1 proteins, including Fos/Jun, to transactivate target genes (18). Thus, it is not surprising, in retrospect, that RANKLinduced osteoclastogenesis involves partnering of Fos/Jun with NFAT2. The fact that Ikeda et al. (1) found that expression of NFAT2 itself is NFAT1/Fos/Jun-dependent is in keeping with the presence of NFAT and AP-1 response elements in the NFAT2 promoter (19). Reflecting its role as an essential RANKL-activated signaling molecule, NFAT2, when overexpressed in wild-type macrophages, prompts osteoclast differentiation in the absence of the osteoclastogenic cytokine (17). RANKL-stimulated gene transcription is, therefore, a reflection of NFAT partnering with Fos/Jun.

Thus, Ikeda et al. (1) provide evidence of important components of RANK-mediated osteoclastogenesis specifically as it pertains to the AP-1 transcription complex. Clearly, TRAF6 is an essential player in RANKLmediated c-Jun and NFAT activation and, perhaps, c-Fos expression. NFAT/Fos/Jun is a critical osteoclastogenic complex, and deletion of any of the three arrests osteoclast formation. The pivotal role that Ikeda et al. document for c-Jun in osteoclast formation is in keeping with the fact that the anti-bone resorptive effects of estrogen are substantially mediated by c-Jun repression (20). Given that RANKL expression is also enhanced in estrogen-deficient women (21), RANKL $\rightarrow$ TRAF6 $\rightarrow$ MAPK kinase 7 $($ MKK7) $\rightarrow$ JNK1 $\rightarrow$ Jun $\rightarrow$ NFAT signaling is likely pivotal to the pathogenesis of postmenopausal osteoporosis, and inhibition of any of the components will theoretically arrest accelerated bone resorption (Figure 1). The therapeutic challenge is how to specifically target these intracellular signaling molecules in osteoclasts.
Address correspondence to: Steven L. Teitelbaum, Department of Pathology and Immunology, Washington University School of Medicine, Campus Box 8118, 660 South Euclid Avenue, St. Louis, Missouri 63110, USA. Phone: (314) 454-8463; Fax: (314) 454-5505; E-mail: teitelbs@path.wustl.edu. 
1. Ikeda, F., et al. 2004. Critical roles of c-Jun signaling in regulation of NFAT family and RANKL-regulated osteoclast differentiation. J. Clin. Invest. 114:475-484. doi:10.1172/JCI200419657.

2. Teitelbaum, S.L. 2000. Bone resorption by osteoclasts. Science. 289:1504-1508.

3. Walker, D.G. 1975. Bone resorption restored in osteopetrotic mice by transplants of normal bone marrow and spleen cells. Science. 190:784-785.

4. Udagawa, N., et al. 1990. Origin of osteoclasts: mature monocytes and macrophages are capable of differentiating into osteoclasts under a suitable microenvironment prepared by bone marrow-derived stromal cells. Proc. Natl. Acad. Sci. U. S. A. 87:7260-7264.

5. Lacey, D.L., et al. 1998. Osteoprotegerin ligand is a cytokine that regulates osteoclast differentiation and activation. Cell. 93:165-176.

6. Yasuda, H., et al. 1998. Osteoclast differentiation factor is a ligand for osteoprotegerin/osteoclastogenesis-inhibitory factor and is identical to TRANCE/ RANKL. Proc. Natl. Acad. Sci. U. S. A. 95:3597-3602.

7. Lam, J., Nelson, C.A., Ross, F.P., Teitelbaum, S.L., and Fremont, D.L. 2001. Crystal structure of TRANCE/ RANKL cytokine reveals determinants of receptor-ligand specificity. J. Clin. Invest. 108:971-980.
doi:10.1172/JCI200113890.

8. Lam, J., et al. 2000. TNF- $\alpha$ induces osteoclastogenesis by direct stimulation of macrophages exposed to permissive levels of RANK ligand. J. Clin. Invest. 106:1481-1488.

9. Li, J., et al. 2000. RANK is the intrinsic hematopoietic cell surface receptor that controls osteoclastogenesis and regulation of bone mass and calcium metabolism. Proc. Natl. Acad. Sci. U. S. A. 97:1566-1571.

10. Boyle, W.J., Simonet, W.S., and Lacey, D.L. 2003 Osteoclast differentiation and activation. Nature. 15:337-342.

11. Grigoriadis, A.E., et al. 1994. c-Fos: a key regulator of osteoclast-macrophage lineage determination and bone remodeling. Science. 266:443-448.

12. Lomaga, M.A., et al. 1999. TRAF6 deficiency results in osteopetrosis and defective interleukin-1, CD40, and LPS signaling. Genes Dev. 13:1015-1024.

13. Naito, A., et al. 1999. Severe osteopetrosis, defective interleukin-1 signalling and lymph node organogenesis in TRAF6-deficient mice. Genes Cells. 4:353-362.

14. David, J.-P., Sabapathy, K., Hoffmann, O., Idarraga, M.H., and Wagner, E.F. 2002. JNK1 modulates osteoclastogenesis through both c-Jun phosphorylationdependent and -independent mechanisms. J. Cell. Sci.
115:4317-4325

15. Kenner, L., et al. 2004. Mice lacking JunB are osteopenic due to cell-autonomous osteoblast and osteoclast defects. J. Cell Biol. 164:613-623.

16. Matsuo, K., et al. 2000. Fosl1 is a transcriptional target of c-Fos during osteoclast differentiation. Nat. Genet. 24:184-187.

17. Takayanagi, H., et al. 2002. Induction and activation of the transcription factor NFATc1 (NFAT2) integrate RANKL signaling in terminal differentiation of osteoclasts. Dev. Cell. 3:889-901.

18. Macian, F., Lopez-Rodriguez, C., and Rao, A. 2001. Partners in transcription: NFAT and AP-1. Oncogene. 20:2476-2489.

19. Zhou, B., et al. 2002. Regulation of the Murine Nfatc1 Gene by NFATc2. J. Biol. Chem. 277:10704-10711.

20. Srivastava, S., et al. 2000. Estrogen decreases osteoclast formation by down-regulating receptor activator of NF-kappa B ligand (RANKL)-induced JNK activation. J. Biol. Chem. 276:8836-8840. doi:10.1074/jbc.M010764200.

21. Eghbali-Fatourechi, G., et al. 2003. Role of RANK ligand in mediating increased bone resorption in early postmenopausal women. J. Clin. Invest. 111:1221-1230. doi:10.1172/JCI200317215.

\title{
AMP-activated protein kinase: the guardian of cardiac energy status
}

\author{
D. Grahame Hardie
}

Division of Molecular Physiology, Faculty of Life Sciences, Wellcome Trust Biocentre, University of Dundee, Dundee, United Kingdom.

\begin{abstract}
Several years ago it was proposed that the AMP-activated protein kinase cascade might protect cells against stresses that deplete cellular ATP. Young et al. have now directly tested this by studying the effects of ischemia and reperfusion in perfused hearts from mice expressing a dominant-negative mutant that suppresses the kinase activity in cardiac muscle (see the related article beginning on page 495). Compared with control hearts, the mutant hearts showed clear evidence for increased necrotic damage and increased apoptosis. These findings may have implications for the treatment of ischemic heart disease.
\end{abstract}

AMP-activated protein kinase (AMPK) is the downstream component of a protein kinase cascade that is highly conserved in all eukaryotic cells (1). AMPK is activated by the rising cellular AMP that (due to the action of adenylate kinase) always $\mathrm{ADP}$ ratio, and this activation is antagonized by high concentrations of ATP (Figure 1). Downstream targets and processes regulated by the kinase are being identified on a regular basis (Figure 2).

Nonstandard abbreviations used: 5-aminoimidazole4-carboxamide riboside monophosphate (ZMP); AMPactivated protein kinase (AMPK).

Conflict of interest: The author has declared that no conflict of interest exists.

Citation for this article:

J. Clin. Invest. 114:465-468 (2004).

doi:10.1172/JCI200422683. accompanies a fall in the cellular ATP/
In general, AMPK switches off ATP-consuming processes such as biosynthetic pathways, while switching on catabolic processes that generate ATP, including cellular uptake of glucose (2) and fatty acids (3) and increased fatty acid oxidation (4) in the heart.

The first evidence that AMPK was activated by metabolic stresses appeared 13 years ago, when my group found that AMPK was activated by ATP depletion caused by incubation of isolated rat hepatocytes with high fructose (5), while Witters et al. (6) reported that it was activated by various metabolic poisons in hepatoma cells. We proposed at the time

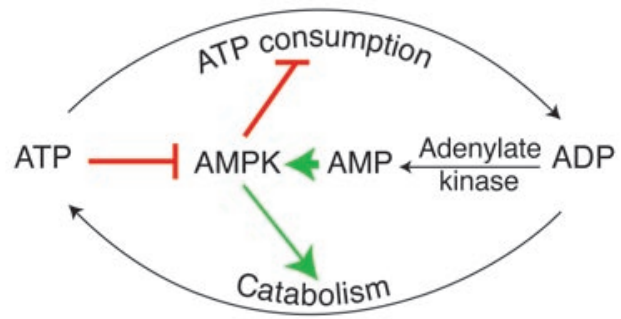

Figure 1

Role of AMPK in regulating energy balance at the single-cell level. The way in which the AMPK system controls the balance between ATP consumption (e.g., by biosynthesis, cell growth, or muscle contraction) and ATP production via catabolism is illustrated. If the rate of ATP consumption exceeds its rate of production, ADP will tend to rise and be converted to AMP by the enzyme adenylate kinase. The rise in level of the activating ligand AMP, coupled with the fall in level of the inhibitory nucleotide ATP, activates AMPK, which then switches off ATP-consuming processes and switches on catabolism in an attempt to redress the balance. 